10.2478/amb-2014-0001

\title{
ASSESSMENT OF BETA-CELL FUNCTION DURING PREGNANCY AND AFTER DELIVERY
}

\author{
M. P. Genova ${ }^{1}$, K. Todorova-Ananieva ${ }^{2}$, B. Atanasova ${ }^{1}$ and K. Tzatchev ${ }^{1}$ \\ ${ }^{1}$ Department of Clinical Laboratory and Clinical Immunology, Medical University, \\ University Hospital "Alexandrovska", Sofia, Bulgaria \\ 2University Clinic of Endocrinology, Medical University, Pleven, Bulgaria
}

Summary. The aim of the present study was to assess $\beta$-cell function using homeostasis model ( $\mathrm{HOMA}-\mathrm{B})$ and disposition index $(\mathrm{DI})$ in pregnant women with/without gestational diabetes, and after delivery. A total of 102 pregnant women between 24-28 gestational weeks (53 with gestational diabetes mellitus (GDM) and 49 with normal glucose tolerance (NGT) and 22 GDM postpartum women (8-12 weeks after delivery) were included in the study. All postpartum women had a history of GDM. HOMAindexes (insulin resistance-HOMA-IR and HOMA-B for assessing $\beta$-cell function) were calculated from fasting glucose and insulin concentrations. To estimate insulin secretion independent of insulin sensitivity, DI was calculated using glucose and insulin levels at 0 and 60 min during the course of a $2 \mathrm{~h} 75 \mathrm{~g}$ oral glucose tolerance test (OGTT). In GDM pregnant women HOMA-B was significantly lower compared to NGT women $(p=0.017)$, but there was no significant difference compared to women after birth (NS). There was difference between NGT and postpartum women $(p<0.05)$. DI was significantly lower for GDM pregnant women in comparison to NGT and postpartum women $(p<0.0001 ; p=0.011)$, between NGT and women after birth $(p<0.04)$. In our study, comparison of HOMA-B in NGT and GDM pregnant women demonstrated that the OR of developing GDM was $0.989(95 \% \mathrm{Cl}, 0.980-0.998, \mathrm{P}=0.013)$, and comparison of $\mathrm{Dl}$ in healthy pregnant and GDM showed that the OR of developing GDM was $0.967(95 \% \mathrm{Cl}, 0.947-0.988, \mathrm{P}=$ 0.002 ). Therefore, HOMA-B and DI appear to be protective factors in the risk of developing GDM. According to our results, assessment of $\beta$-cell function, using HOMA-B and DI, showed that they are lower in GDM than NGT group and postpartum women. It is important to note that HOMA-B did not show significant difference between GDM pregnant and women after delivery with a history for GDM. We assume that pregnant women with GDM have a pancreatic $\beta$-cell defect that remains after birth. These women are at increased risk for developing diabetes mellitus, the most frequent type 2 diabetes, in the future after birth.

Key words: gestational diabetes mellitus, $\beta$-cell function, homeostasis model assessment, disposition index 


\section{INTRODUCTION}

$\mathrm{G}$ estational diabetes mellitus (GDM) is carbohydrate intolerance that begins or is first recognized during pregnancy [19]. The underlying mechanisms for the development of GDM are related to $\beta$-cell dysfunction and insulin resistance or decreased maternal insulin sensitivity. Pregnancy is normally characterized by progressive insulin resistance that begins near midpregnancy and progresses through the third trimester to levels that approximate the insulin resistance typical for type 2 diabetes [1]. Ryan et al. were the first to report a $40 \%$ decrease in insulin sensitivity and $67 \%$ reduction in relation to insulin resistance in women with GDM compared with a pregnant control group in late pregnancy using the euglycemic clamp [25].

Pancreatic $\beta$-cells normally increase their insulin secretion during pregnancy to compensate for the insulin resistance. As a result, changes in circulating glucose levels over the course of pregnancy are quite small in comparison to the large changes in insulin sensitivity. Thus, robust plasticity of $\beta$-cell function in the face of progressive insulin resistance is the hallmark of normal glucose regulation during pregnancy. During pregnancy with GDM, the pancreatic $\beta$-cell function is insufficient to meet the body's insulin needs. Existing evidence suggests that $\beta$-cell defects in GDM result from the same spectrum of causes that underlie hyperglycemia [6]. Different approaches reveal large defect in pancreatic $\beta$-cell function in women with GDM both during and after pregnancy $[16,24,4]$. The defect can be quantified by expressing insulin levels relative to each individual's degree of insulin resistance, using the hyperbolic relationship between insulin sensitivity and insulin secretion $[3$, 17]. The product of these two variables, referred to as the disposition index (DI), can be calculated and has showed the inability of the $\beta$-cell to compensate for insulin resistance [7]. The DI declines well before glucose levels rise into the diabetic range. Thus, a low DI is pointed as a marker of inadequate $\beta$-cell compensation [27].

The aim of the current study was to assess $\beta$-cell function using homeostasis model and disposition index in pregnant women with/without gestational diabetes, and postpartum.

\section{MATERIAL AND METHODS}

A total of 102 pregnant women between 21-28 gestational weeks (53 with GDM and 49 with normal glucose tolerance (NGT) and 22 postpartum women (8-14 weeks) were included in the study. We assessed selected anthropometric, clinical, and pathophysiological parameters in all of them. Exclusion criteria for pregnant women were chronic diseases, acute infection during pregnancy or establishing of diagnosis, drugs that are known to affect the carbohydrate metabolism or interfere with insulin sensitivity, multiple pregnancies, known diabetes, fetal malformation, or other severe maternal illnesses, age $<18$ or $>45$ years. 
All the tested pregnant women with no previously diagnosed diabetes went through screening for GDM with a $2 \mathrm{~h} 75 \mathrm{~g}$ oral glucose tolerance test (OGTT) between 21 and 28 weeks of gestation. Diagnosis of GDM was established in accordance with the recommendations of the International Diabetes in Pregnancy Study Group - fasting plasma glucose $\geq 5.1 \mathrm{mmol} / \mathrm{L}, 1 \mathrm{~h} \geq 10.0 \mathrm{mmol} / \mathrm{L}$, or $2 \mathrm{~h} \geq 8.5 \mathrm{mmol} / \mathrm{L}$ [14]. The OGTT stratified participants into two glucose tolerance groups: normal glucose tolerance (NGT) and gestational diabetes mellitus (GDM). All women after delivery ( $11 \pm 3.4$ weeks) with a history of GDM were screened with a $2 \mathrm{~h} 75 \mathrm{~g}$ oral glucose tolerance test.

The following data were collected for all women: age, pregnancy BMI at GDM diagnosis, BMI after delivery, gestational weeks, weeks postpartum. Samples were drawn just before and at 60 min after ingestion of $75 \mathrm{~g}$ glucose for GDM diagnosis and for screening after birth.

Blood samples for insulin and glucose measurements were drawn from individuals in a fasting state between 8.00 am and $9.00 \mathrm{am}$, after a 12-hour fasting pause overnight. Plasma glucose was determined in the venous blood by the method of oxygen consumption (Analox GM9, Analox Instruments USA, reference range 2.8-6.1 $\mathrm{mmol} / \mathrm{L}$ ). Serum insulin concentration in the venous blood was analysed by electrochemiluminescent immunoassay (ECLIA) (Elecsys 2010, Roshe Diagnostics, reference range $2.6-24.9 \mu \mathrm{U} / \mathrm{ml}$ ).

All laboratory assays were performed at Central Clinical Laboratory, University Hospital "Alexandrovska".

HOMA-IR values were calculated from the concentrations of insulin and glucose using the following formula: fasting serum insulin $(\mu \mathrm{U} / \mathrm{ml}) \times$ fasting plasma glucose $(\mathrm{mmol} / \mathrm{l}) / 22.5$. HOMA-B was calculated using the following formula: $20 \times$ fasting insulin $(\mu \mathrm{lU} / \mathrm{ml}) /$ fasting glucose $(\mathrm{mmol} / \mathrm{ml})-3.5$ [18]. To estimate insulin secretion independent of insulin sensitivity, the DI was calculated as the insulinogenic index [(insulin 60-insulin 0)/(glucose 60-glucose 0)] [22] divided by the insulin resistance index [15].

\section{Statistical analysis}

Data were analyzed using Statistical software for Windows 13.0 by SPSS SPSS. The Shapiro-Wilk test was used to determine whether each variable had a normal distribution. These variables were expressed as mean values \pm SD. The Kruskal-Wallis test and the $U$ Mann-Whitney test were used to compare selected groups. A $p<0.05$ value was defined as significant. Comparisons between the subgroups were performed by one-way analysis of variance (ANOVA) with post-hoc analysis to locate the differences.

\section{RESULTS}

A total of 102 pregnant women were enrolled in the study (mean age $31.5, S D \pm$ 4.55 years) and divided in two main groups based on the plasma glucose concentration and the IAPSDG criteria for diagnosis of GDM: one group $(n=49)$ of subjects with NGT and second group with impaired glucose tolerance - pregnant with GDM $(n=53)$. Wom- 
en in the NGT group were younger than those in the GDM group and the postpartum group with statistically significant difference $(p<0.003, p<0.01)$. BMI was significantly higher in GDM patients compared to NGT and women postpartum $(p<0.011 ; p<0.001)$. Meanwhile, BMI was higher in women after delivery compared to NGT $(p<0.0001)$ (Table 1). Three groups were comparable regarding the fasting glucose, glucose at 60 min, fasting insulin, insulin at 60 min, HOMA-IR, HOMA-B and DI (Table 2).

Table 1. Clinical characteristics of the participants

\begin{tabular}{|l|c|c|c|c|}
\hline Characteristics & $\begin{array}{c}\text { NGT group } \\
\text { (n1 = 49) }\end{array}$ & $\begin{array}{c}\text { GDM group } \\
\text { (n2 = 53) }\end{array}$ & $\begin{array}{c}\text { After delivery } \\
\text { (n3 = 22) }\end{array}$ & $\begin{array}{c}\text { Statistical } \\
\text { significance }\end{array}$ \\
\hline Age (years) & ${ }^{*} 30,51 \pm 4,72 \bullet$ & ${ }^{*} 32.6 \pm 4,39$ & $\bullet \cdot 33.6 \pm 4.3$ & $\begin{array}{c}\mathrm{p}<0.003^{*} \\
\mathrm{p}<0.01 \cdot \bullet\end{array}$ \\
\hline BMl (kg/m2) & ${ }^{* *} 26.83 \pm 5,54^{*}$ & ${ }^{*} 30.56 \pm 6.9^{*}$ & ${ }^{*} 27.9 \pm 6,27^{* *}$ & $\begin{array}{c}\mathrm{p}<0,011^{*} \\
\mathrm{p}<0,001^{*} \\
\mathrm{p}<0,0001^{* *}\end{array}$ \\
\hline Gestational weeks & $24 \pm 4$ & $24 \pm 4$ & - & $\mathrm{NS}$ \\
\hline Metabolic syndrome & $\mathrm{n} 1=6(12,2 \%)$ & $\mathrm{n} 2=13(24,5 \%)$ & $\mathrm{n} 3=4(18.1 \%)$ & \\
\hline
\end{tabular}

GDM - gestational diabetes mellitus; BMI - body mass index. Values of parameters are presented as mean \pm SD or count (percentage); T1DM-Type 1 Diabetes Mellitus; T2DM-Type 2 Diabetes Mellitus The diagnosis of metabolic syndrome is placed outside glycemic fluctuations

Table 2. Characteristics of the patients' metabolic parameters

\begin{tabular}{|c|c|c|c|c|}
\hline Characteristics & $\begin{array}{c}\text { Pregnant with NGT } \\
(\mathrm{n} 1=49)\end{array}$ & $\begin{array}{c}\text { Pregnant with GDM } \\
(\mathrm{n} 2=53)\end{array}$ & $\begin{array}{c}\text { After delivery } \\
\text { (n3 = 22) }\end{array}$ & $\begin{array}{c}\text { Statistical } \\
\text { significance }\end{array}$ \\
\hline Fasting glucose $(\mathrm{mmol} / \mathrm{l})$ & $\cdot 4.62 \pm 0.28^{*}$ & $5.93 \pm 1.04^{*}$ & $\cdot 5.33 \pm 0.79$ & $\begin{array}{l}p<0,0001^{*} \\
p<0.0001 .\end{array}$ \\
\hline $\begin{array}{l}\text { Glucose at } 60 \mathrm{~min} \\
(\mathrm{mmol} / \mathrm{l})\end{array}$ & ${ }^{*} 6.95 \pm 1.37^{* *}$ & $+8.49 \pm 2.29^{* \star}$ & ${ }^{*} 7.62 \pm 2.36+$ & $\begin{array}{c}p<0.001^{*} \\
p=0.0004^{*} \\
p=0.002+\end{array}$ \\
\hline Fasting insulin (mIU/l) & $* 11.35 \pm 4.98^{* *}$ & $\bullet \cdot 13.84 \pm 8.43^{* *}$ & $\bullet \cdot 10.6 \pm 7.53^{*}$ & $\begin{array}{l}p=0.02^{* *} \\
p<0.01 . \bullet \\
p<0.01^{*}\end{array}$ \\
\hline $\begin{array}{l}\text { Insulin at } 60 \mathrm{~min} \\
(\mathrm{mlU} / \mathrm{l})\end{array}$ & ${ }^{*} 63.89 \pm 32.93^{\star \star *}$ & $\cdot 76.98 \pm 57.47^{\star \star *}$ & ${ }^{*} 68.47 \pm 54.76 \bullet$ & $\begin{array}{l}p<0.011^{* * *} \\
p=0.0048^{*} \\
p=0,0021\end{array}$ \\
\hline HOMA-IR & ${ }^{*} 1.79 \pm 1.08 \dagger \dagger$ & $\dagger 3.8 \pm 3.05 \dagger \dagger$ & $\dagger 2.5 \pm 1.94^{*}$ & $\begin{array}{c}p<0.0001 \dagger \dagger \\
p=0.017 \dagger \\
p=0.004^{*}\end{array}$ \\
\hline HOMA-B & $• 126.48 \pm 57.69 \bullet \bullet$ & $\cdot 99.94 \pm 41.51^{*}$ & ${ }^{*} 99.41 \pm 41.37 \cdot \bullet$ & $\begin{array}{c}P<0.05 \bullet \\
p=0.017 \\
N^{*}\end{array}$ \\
\hline Disposition index & $\cdot 13.22 \pm 21.70^{*}$ & ${ }^{* *} 6.47 \pm 12.93^{*}$ & ${ }^{* *} 10.06 \pm 15.50$ & $\begin{array}{c}p<0.0001^{*} \\
p=0.011^{* *} \\
p<0.04\end{array}$ \\
\hline
\end{tabular}




\section{DISCUSSION}

Pancreatic beta-cell dysfunction is one of the main pathogenetic mechanisms of GDM [13]. Women with GDM have a chronic defect in $\beta$-cell function [5]. Although this defect likely precedes the pregnancy [2], it is first detected clinically in the form of insufficient $\beta$-cell compensation for insulin resistance in late pregnancy. The $\beta$-cell defect in women with GDM is still present in the postpartum period [8].

The easiest and most popular assessment of $\beta$-cell function is the homeostatic index HOMA-B. It is widely used because of its simplicity and reflects the release of insulin under nonstimulated conditions [9].

The first important observation resulting from our study is that significant differences were found in the studied population of women. GDM women were more insulin resistant (HOMA-IR) and their $\beta$-cell function was not able to meet the insulin demands induced by the pregnancy, in comparison to NGT and postpartum groups ( $p<0.0001, p=0.017$ ). Secondly, according to our results, assessment of $\beta$-cell function, using HOMA-B and DI, showed that they are lower in GDM than NGT group and postpartum women. It is important to note that HOMA-B did not show significant difference between GDM pregnant and women after delivery with a history for GDM. Finally, young mothers were more insulin-resistant in comparison to NGT $(p=0.004)$. It is important to note that the value of HOMA-IR remained high during this period after birth. Morkrid et al. (2012) established that the GDM women were more insulin resistant compared with the GDM women 3 months post partum, measured by HOMA-IR, a finding that is opposite to our results. They found that $\beta$-cell function, measured by HOMA- $\beta$, was similar in these groups [21], as opposed to our results. HOMA-B was statistically lower in GDM pregnancy than NGT group $(p=0.017)$. This trend was also observed in young mothers with a history of GDM when compared to GDM pregnant regarding HOMA-B (NS). The reduced insulin secretion in the period after birth is normal and is expressed by reliable difference in HOMA-B index between the NGT group and women post partum $(p<0.05)$. No significant difference for HOMA-B between GDM pregnant women and those after delivery support the hypothesis that beta cell defect continues during the postpartum period. Endo et al. (2006) found higher values of HOMA-B in pregnant women with NGT compared to pregnant with GDM $(p<0.01)$ [11]. A similar result reported Das S. and Behera M. K. et al. (2010) who observed that HOMA-B has a statistically significant higher value in pregnant women with NGT in comparison with GDM $(p<0.01)[10]$.

Beta-cell secretory function can be assessed by use of the oral disposition index. DI was computed as a composite measure of $\beta$-cell function. In our study DI decreased progressively from NGT to GDM $(p<0.0001)$ and it was significantly higher, compared to women after delivery $(p<0.04)$. Significant difference was observed between pregnant with GDM and young women after birth $(p=0.011)$. Women after delivery exhibited a higher absolute value for DI compared to pregnant women with GDM, but it remained significantly lower than the value in the healthy pregnant women group. Prospective studies indicate that DI decrease before increasing the 
glucose level to values typical of diabetes. So, low level of DI has been reported to be an early marker of inadequate $\beta$-cell compensation [26].

Qvigstad et al. (2010) found lower values for DI in GDM pregnant women compared to NGT women [23]. Similar results obtained Miyakoshi et al. (2011). They estimated $\beta$-cell function in pregnant women with GDM (according to the criteria of IAPDSG-2010), using DI, obtained during the $75 \mathrm{~g}$ OGTT. They found that in pregnant with GDM DI was significantly lower compared to healthy pregnant women $(p<0.001)$. The results suggest that pregnant women with GDM, identified under the new consensus criteria, have a greater risk of developing diabetes type 2 in the future, and adverse perinatal outcomes [20].

In our study, comparison of HOMA-B in NGT and GDM pregnant women demonstrated that the OR of developing GDM was $0.989(95 \% \mathrm{Cl}, 0.980-0.998$, $\mathrm{P}=0.013$ ), and comparison of $\mathrm{DI}$ in healthy pregnant and GDM showed that the OR of developing GDM was $0.967(95 \% \mathrm{Cl}, 0.947-0.988, \mathrm{P}=0.002)$. Therefore, HOMA$\mathrm{B}$ and $\mathrm{DI}$ appear to be protective factors in the risk of developing GDM.

Such differences in HOMA-B and DI within the three selected groups, indicate a significant pathophysiological variation in the severity of $\beta$-cell dysfunction at the time of GDM diagnosis and after birth. The precise mechanisms of $\beta$-cells mass expansion during human pregnancy have only partially been elucidated [12].

In the interpretation of the lower values of the markers of beta-cell function HOMA-B and DI in GDM pregnant and postpartum women, one should take into consideration the older age $(p<0.003, p<0.01)$, higher gestational $\mathrm{BMI}(\mathrm{p}<0,011$, $p<0,0001)$, higher level of HOMA-IR $(p<0.0001, p=0.004)$ and pregnancy - induced metabolic alterations, which reveal the tendency towards a worse function of these cells with age, already seen even in a younger population.

In conclusion, we established reduced $\beta$-cell function in pregnancy with GDM and 3 month post partum in comparison with NGT pregnant women, using HOMA-B and DI. These women are at increased risk of developing diabetes mellitus, the most frequent type 2 diabetes, in the future time after birth.

\section{REFERENCES}

1. B a rb ou r, L. A. et al. Cellular mechanisms for insulin resistance in normal pregnancy and gestational diabetes. - Diabetes Care, 30, 2007, (Suppl. 2), S112-S119.

2. B a s u, R. et al. Mechanisms of age-associated determination in glucose tolerance, contribution of alterations in insulin secretion, action and clearance. - Diabetes, 52, 2003, 1738-1748.

3. B ergman, R. N., L. S. Phillips et C. Cobelli. Physiologic evaluation of factors controlling glucose tolerance in man: measurement of insulin sensitivity and $\beta$-cell glucose sensitivity from the response to intravenous glucose. - J. Clin. Invest., 68, 1981, № 68, 1456-1467.

4. Bu ch an an, T. A. Pancreatic B-cell defects in gestational diabetes: implications for the pathogenesis and prevention of type 2 diabetes. - J. Clin. Endocrinol. Metab., 86, 2001, 989-993. 
5. Buchanan, T. A. et A. H. Xiang. Gestational diabetes mellitus. - J. Clin. Invest., 115, 2005, 485-491.

6. B u ch a n a n, A. T., A. H. Xiang, L. K. Siri et R. Watanabe. What is gestational diabetes? - Diabetes Care., 30, 2007, № 2, 105-111.

7. Buchanan, T. A. et al. Preservation of pancreatic $\beta$-cell function and prevention of type 2 diabetes by pharmacological treatment of insulin resistance in high-risk Hispanic women. - Diabetes, 51, 2002, 2796-2803.

8. Cata la no, P. M. et al. Longitudinal changes in glucose metabolism during pregnancy in obese women with normal glucose tolerance and gestational diabetes mellitus. - Am. J. Obstet. Gynecol., 180, 1999, 903-916.

9. Co belli, C. et al. Assessment of $\beta$-cell function in humans, simultaneously with insulin sensitivity and hepatic extraction, from intravenous and oral glucose tests. - Am. J. Physiol. Endocrinol. Metab., 293, 2007, E1-E15.

10. Das , S. M., S. Misra et A. Baliarsihna. Beta-cell function and insulin resistance in pregnancy and their relation to fetal development. - Metab. Syndr. Relat. Disord., 8, 2010, № 1, 25-32.

11. Endo, S. et al. Differences in insulin sensitivity in pregnant women with overweight and gestational diabetes mellitus. - Gynecological Endocrinology, 22, 2006, № 6, 343-349.

12. Ernst, S. et al. Mechanisms in the adaptation of maternal $\beta$-cells during pregnancy. - Diabetes Management, 1, 2011, 239-248.

13. Hom ko, C. et al. Guenther. Insulin secretion during and after pregnancy in patients with gestational diabetes mellitus. - J. Clin. Endocrinol. Metab., 86, 2001, 568-573.

14. Internatio nal Association of Diabetes and Pregnancy Study Groups Consensus Panel 2010. Diabetes Care, 33, 2010, № 3, 676-682.

15. $\mathrm{Kahn}, \mathrm{S}$. E. Clinical review 135: the importance of $\beta$-cell failure in the development and progression of type 2 diabetes. - J. Clin. Endocrinol. Metab., 86, 2001, 4047-4058.

16. $\mathrm{Kahn}, \mathrm{S}$. E. The relative contributions of insulin resistance and beta-cell dysfunction to the pathophysiology of type 2 diabetes. - Diabetologia, 46, 2003, 3-19.

17. Ka h n, S. E. et al. Quantification of the relationship between insulin sensitivity and B-cell function in human subjects: evidence for a hyperbolic function. - Diabetes, 42, 1993, 1663-1672.

18. Matthew S, D. R, J. P Hosker et A. S Rudenski. Homeostasis model assessment: insulin resistance and beta cell function from fasting plasma glucose and insulin concentrations in man. - Diabetologia, 28, 1985, 412-419.

19. Metzger, B. E. et D. R. Coustan. Summary and recommendations of the Fourth International Workshop Conference on Gestational Diabetes Mellitus. - Diabetes Care, 21, 1998, (Suppl. 2), B161-B167.

20. Miyakoshi, K. et al. Pancreatic $\beta$-cell Function in Women With Gestational Diabetes Mellitus Defined by New Consensus Criteria. - Diabetes Care, 34, 2011, 1-8.

21. Mørkrid, K. et al. Failure to increase insulin secretory capacity during pregnancy-induced insulin resistance is associated with ethnicity and gestational diabetes. - Eur. J. Endocrinol., 167, 2012, 579-588.

22. Phillips, D. I., P. M. Clark, C. N. Hales et C. Osmond. Understanding oral glucose tolerance: comparison of glucose or insulin measurements during the oral glucose tolerance test with specific measurements of insulin resistance and insulin secretion. - Diabet. Med., 11, 1994, 286-292.

23. Qvigstad, E. et al. Overweight is associated with impaired $\beta$-cell function during pregnancy: a longitudinal study of 553 normal pregnancies. - Eur. J. Endocrinol., 162, 2010, 67-73.

24. Ryan, E. A. et al. Defects in insulin secretion and action in women with a history of gestational diabetes. - Diabetes, 44, 1995, $506-512$. 
25. Ry a n, E. A., M. J. O'Sullivan et J. Skyler. Insulin action during pregnancy. Studies with the euglycemic clamp technique. - Diabetes, 34, 1985, 380-389.

26. Utzschneider, K. et al. Oral Disposition Index Predicts the Development of Future Diabetes Above and Beyond Fasting and 2-h Glucose Levels. - Diabetes Care, 32, 2009, № 2, 335-341.

27. Xiang, A. $\mathrm{H}$. et al. Coordinate changes in plasma glucose and pancreatic $\beta$-cell function in Latino women at high risk for type 2 diabetes. - Diabetes, 55, 2006, 1074-1079.

Corresponding author:

Mariana P. Genova, MD, PhD

Department of Clinical Laboratory and Clinical Immunology

Medical University

University Hospital "Alexandrovska"

1 Sv. Georgy Sofiyski st.

$\mathrm{Bg}-1431$ Sofia

e-mail: mariana8sofia@yahoo.com 